\title{
THE
}

\section{Improvements to the methods used to measure bubble attenuation using an underwater acoustical resonator}

\author{
Helen Czerski \\ University of Rhode Island \\ Svein Vagle \\ David M. Farmer \\ University of Rhode Island, farmerd@uri.edu \\ Nick Hall-Patch
}

Follow this and additional works at: https://digitalcommons.uri.edu/gsofacpubs

Terms of Use

All rights reserved under copyright.

\section{Citation/Publisher Attribution}

Czerski, H., Vagle, S., Farmer, D. M., \& Hall-Patch, N. (2011). Improvements to the methods used to measure bubble attenuation using an underwater acoustical resonator. Acoustical Society of America, 130(5), 3421-3430. doi: 10.1121/1.3569723

Available at: https://doi.org/10.1121/1.3569723

This Article is brought to you for free and open access by the Graduate School of Oceanography at DigitalCommons@URI. It has been accepted for inclusion in Graduate School of Oceanography Faculty Publications by an authorized administrator of DigitalCommons@URI. For more information, please contact digitalcommons-group@uri.edu. 


\section{Improvements to the methods used to measure bubble attenuation using an underwater acoustical resonator}

Helen Czerski, Svein Vagle, David M. Farmer, and Nick Hall-Patch

Citation: The Journal of the Acoustical Society of America 130, 3421 (2011); doi: 10.1121/1.3569723

View online: https://doi.org/10.1121/1.3569723

View Table of Contents: http://asa.scitation.org/toc/jas/130/5

Published by the Acoustical Society of America

\section{Articles you may be interested in}

Reverberation effects in acoustical resonators used for bubble measurements

The Journal of the Acoustical Society of America 118, 2954 (2005); 10.1121/1.2047148

The effect of coupling on bubble fragmentation acoustics

The Journal of the Acoustical Society of America 129, 74 (2011); 10.1121/1.3514416

Model calculations of the underwater noise of breaking waves and comparison with experiment

The Journal of the Acoustical Society of America 127, 3394 (2010); 10.1121/1.3419774

A mechanism stimulating sound production from air bubbles released from a nozzle

The Journal of the Acoustical Society of America 123, EL126 (2008); 10.1121/1.2908198

Waveguide propagation of ambient sound in the ocean-surface bubble layer

The Journal of the Acoustical Society of America 86, 1897 (1989); 10.1121/1.398568

Review of scattering and extinction cross-sections, damping factors, and resonance frequencies of a spherical gas bubble

The Journal of the Acoustical Society of America 130, 3184 (2011); 10.1121/1.3628321 


\title{
Improvements to the methods used to measure bubble attenuation using an underwater acoustical resonator
}

\author{
Helen Czerskia) \\ Graduate School of Oceanography, University of Rhode Island, South Ferry Road, Narragansett, \\ Rhode Island 02882 \\ Svein Vagle \\ Institute of Ocean Sciences, Fisheries and Oceans Canada, Sidney, British Columbia, Canada V8L 4B2 \\ David M. Farmer \\ Graduate School of Oceanography, University of Rhode Island, South Ferry Road, Narragansett, \\ Rhode Island 02882 \\ Nick Hall-Patch \\ Institute of Ocean Sciences, Fisheries and Oceans Canada, Sidney, British Columbia, Canada V8L 4B2
}

(Received 2 July 2010; revised 22 February 2011; accepted 25 February 2011)

\begin{abstract}
Active acoustic techniques are commonly used to measure oceanic bubble size distributions, by inverting the bulk acoustical properties of the water (usually the attenuation) to infer the bubble population. Acoustical resonators have previously been used to determine attenuation over a wide range of frequencies $(10-200 \mathrm{kHz})$ in a single measurement, corresponding to the simultaneous measurement of a wide range of bubble sizes (20-300 $\mu$ m radii). However, there is now also considerable interest in acquiring measurements of bubbles with radii smaller than $16 \mu \mathrm{m}$, since these are thought to be important for ocean optics and as tracers for near-surface flow. To extend the bubble population measurement to smaller radii, it is necessary to extend the attenuation measurements to higher frequencies. Although the principles of resonator operation do not change as the frequency increases, the assumptions previously made during the spectral analysis may no longer be valid. In order to improve the methods used to calculate attenuation from acoustical resonator outputs, a more complete analysis of the resonator operation is presented here than has been published previously. This approach allows for robust attenuation measurements over a much wider frequency range and enables accurate measurements from lower-quality spectral peaks.
\end{abstract}

(C) 2011 Acoustical Society of America. [DOI: 10.1121/1.3569723]

PACS number(s): 43.30.Gv, 43.20.Ks [NPC]

Pages: $3421-3430$

\section{INTRODUCTION}

Although the research presented in this paper is motivated entirely by the need to improve oceanic bubble measurements, the subject matter is more general: the challenge of making very localized and accurate measurements of acoustical attenuation in a rapidly changing natural aquatic environment. Upper ocean acoustic attenuation measurements have traditionally been made using a single frequency source and one or more distant receivers. ${ }^{1-3}$ The two main drawbacks to this approach for bubble measurements are the need for a long path length to get measureable attenuation and the limited range of bubble sizes that can be measured simultaneously. The internal structure of oceanic bubble plumes is a complex subject and has rarely, if ever, been studied directly. However, it seems likely that these structures are inhomogeneous on size scales of a meter and below, and they are known to contain bubbles from a few microns to a few millimeters in radius. Medwin and co-workers ${ }^{4,5}$ developed the acoustical resonator concept in order to make much more detailed measurements inside oceanic bubble plumes.

\footnotetext{
a) Author to whom correspondence should be addressed. Electronic mail: H.Czerski.97@cantab.net
}

Farmer et al ${ }^{6,7}$ made improvements to the original resonator design and carried out a theoretical analysis of its operation. Since then, resonators have been used regularly ${ }^{8,9}$ to measure the size distributions of bubbles with radii between 16 and $300 \mu \mathrm{m}$, corresponding to an operating frequency range of 5-200 kHz. However, several recent papers have discussed the possible importance of even smaller bubbles for optical scattering, ${ }^{10}$ and the expected Mie scattering from bubbles with radii as low as $0.1 \mu \mathrm{m}$ has been calculated. ${ }^{11}$ The acoustical resonator is a good candidate instrument to observe such bubbles, but to measure of bubbles with a radius of $3 \mu \mathrm{m}$, the resonator needs to be operated at frequencies as high as $1 \mathrm{MHz}$.

The acoustical resonator operation does not change as frequency increases, but the engineering challenges are considerable when the system is operated at these higher frequencies. For example, small imperfections on the transducers, small deviations in their alignment, and electronic issues are increasingly problematic as the acoustic frequency increases. The result of these issues is that the peak quality decreases significantly as the frequency approaches $1 \mathrm{MHz}$. As will be shown, this means that the Lorentzian peak shapes assumed in previous studies are no longer appropriate. The eigenvalue of the resonance (described in 


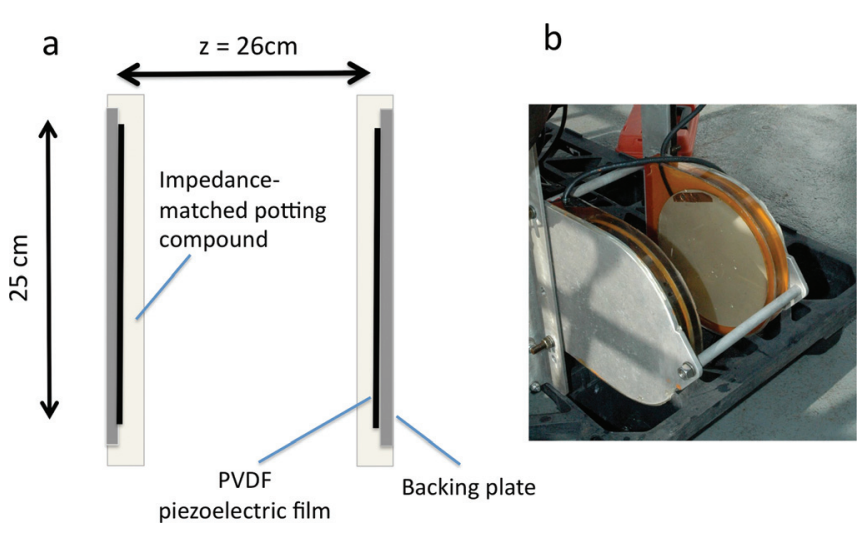

FIG. 1. (Color online) Panel (a) shows a schematic diagram of an acoustical resonator, with approximate dimensions. Panel (b) shows a recent resonator.

Sec. III) is a better measure of the strength of the resonant peak.

The aim of this paper is to introduce a matrix-based analysis of the acoustical resonator, resulting in a better understanding of its spectral response. This leads to a method for interpreting peak heights with a stronger basis in the fundamental resonator physics, which offers a way to deal with the realities of high-frequency resonator designs.

\section{ACOUSTICAL RESONATOR}

Figure 1 shows the basic design of a recent acoustical resonator. It is a relatively simple and mechanically robust instrument, making it suitable for deployments for extended periods in the hostile upper ocean wave zone. The instrument consists of two large flat transducers (approximately $0.25 \mathrm{~m}$ in diameter), made up of metal backing plates covered by a $110 \mu \mathrm{m}$ layer of PVDF (polyvinylidenedifluoride) piezoelectric material coated by a film of $\mathrm{NiCu}$ or $\mathrm{Ag}$. One transducer transmits broadband pseudorandom noise which is reflected between the transducer plates, providing an effective path length many times the physical distance between the transducers. The acoustic pressure field at the receive transducer is digitized, and the power spectral density is calculated for subsequent analysis. The resonator sides are open so that the water between the plates at any given time is representative of the water surrounding the device (Fig. 1). At regular frequency intervals, defined by the transducer spacing, the reflecting waves constructively interfere to generate a resonant peak in the power spectrum. An example is shown in Fig. 2.

The resonant peaks are sensitive to changes in attenuation caused by changes in water properties and the presence of bubbles between the transducer plates. By comparing the peaks from a spectrum measured in a bubble-free environment with the peaks measured in a natural attenuating (bubbly) environment, it is possible to relate changes in peak height to changes in the bulk attenuation of the water at each peak frequency. Figure 3 shows part of a typical power density spectrum, with and without bubbles present. The frequency of any given peak will depend on the speed of sound in the medium between the plates, and is therefore also affected by the presence of bubbles. ${ }^{3,6}$ However, this effect will not be considered in the present analysis.

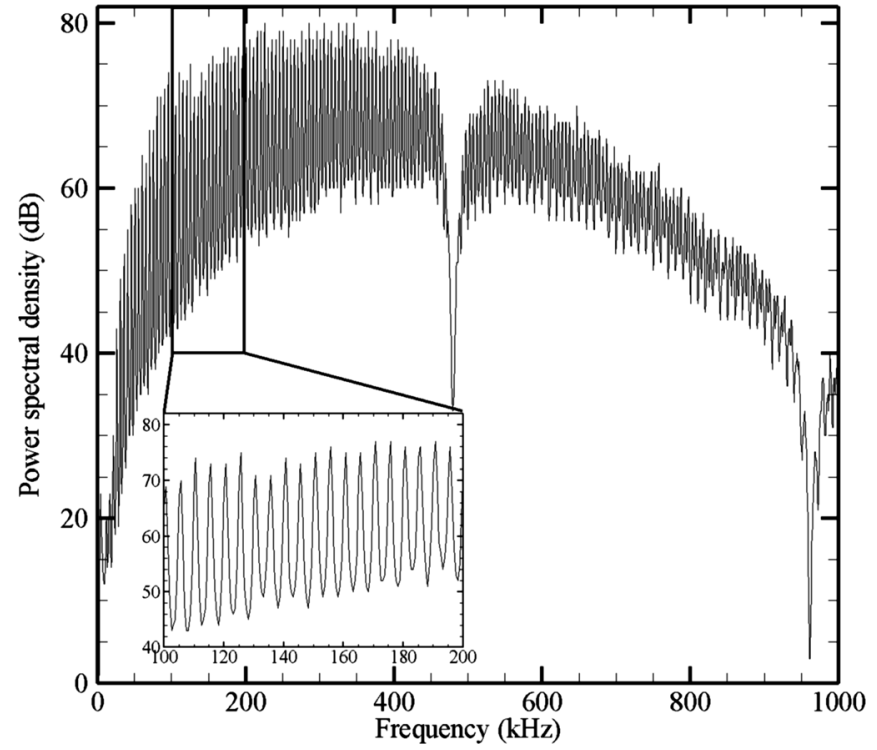

FIG. 2. An example of the noncalibrated power spectral density in decibels measured by a current resonator. The inset shows the spectral peaks at frequencies between 100 and $200 \mathrm{kHz}$. The features at 480 and $960 \mathrm{kHz}$ are due to reflections within the back plate, as described in the text.

The material of the backing plates and their thickness are chosen to maximize the reflection coefficient of the plates, while limiting their weight and the number and location of destructive interference zones (Fig. 2). Typically, we have been using aluminum or mild steel backing plates with thicknesses of either 25.4 or $12.7 \mathrm{~mm}(1 / 2$ or $1 / 4 \mathrm{in}$.). The transducers are covered with a potting compound that has an acoustic impedance equal to that of water to make them waterproof. Internal acoustic reflections inside the backing plate will cancel out the reflected signal at some frequencies, and the spectral regions associated with this cancellation are determined by the combination of the sound speed and the thickness of the plate, as described in Farmer et al. ${ }^{6}$ This can be observed at 480 and $960 \mathrm{kHz}$ in Fig. 2 where the backing plates are made of $12.7 \mathrm{~mm}(1 / 4 \mathrm{in}$.) mild steel. In addition to the choice of backing plate size and material, the most important design considerations are the performance of the transducer (including its electronic components) and how well the potting compound is impedance-matched to the water.

The acoustical resonator is approximately $0.3 \mathrm{~m}$ in size, and the latest resonator design can make a measurement once

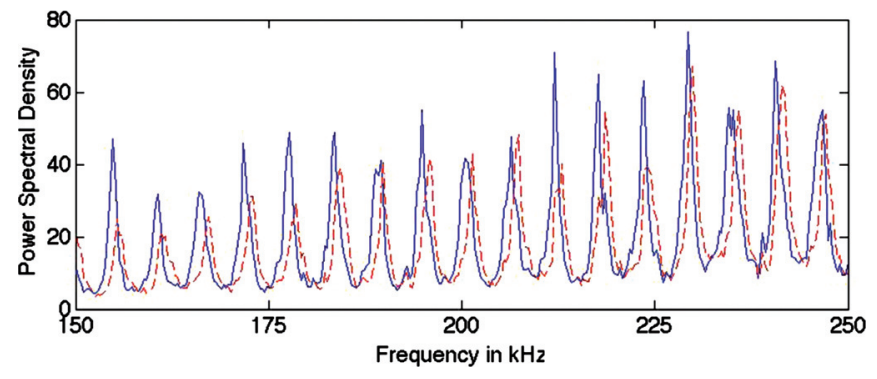

FIG. 3. (Color online) The solid line shows a typical bubble-free spectrum over the frequency range from 150 to $250 \mathrm{kHz}$. The dashed line shows an attenuated spectrum from the same resonator during an ocean deployment. The presence of bubbles reduces the peak height and introduces a phase shift so that the peaks move sideways slightly. 


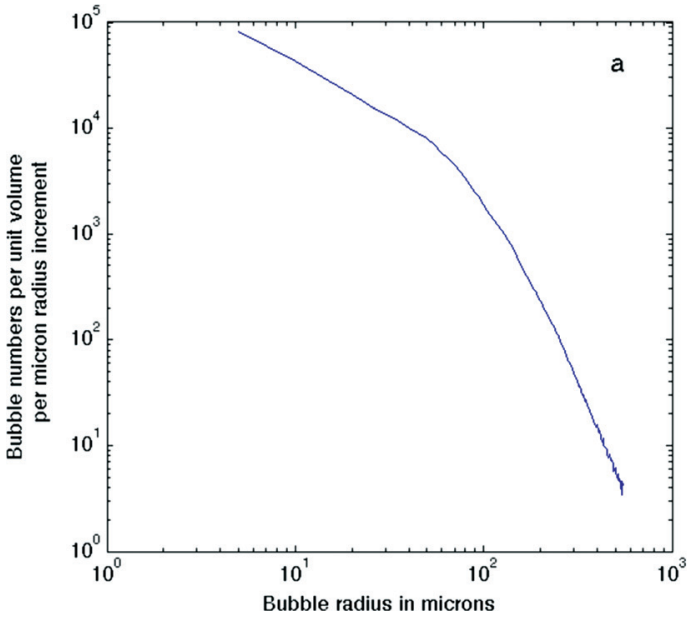

every second, resulting in an excellent temporal and spatial resolution. Another major advantage is that resonators of this size produce resonant peaks approximately every $4 \mathrm{kHz}$, providing good frequency resolution for the attenuation measurements.

Hardware construction is challenging because once the transducer is encased in the potting compound, no further alterations are possible. Testing the transducer extensively before potting is not possible because the transducer is not waterproof at this stage. Development of manufacturing techniques required to produce resonators with a reliable and reproducible response in the $200 \mathrm{kHz}$ to $1 \mathrm{MHz}$ range is ongoing. Regardless of the engineering challenges associated with operating $\mathrm{MHz}$ resonators, the evident loss of fidelity of high-frequency peaks makes it important to understand how to extract data from imperfect spectra.

Another reason for more careful consideration of the higher frequency peaks is that we expect the magnitude of the attenuation due to bubbles to be considerably lower at the higher frequencies. Figure 4 shows a typical oceanic bubble size distribution and the attenuation with frequency that this would cause. The expected attenuation decreases rapidly for frequencies higher than $200 \mathrm{kHz}$, and consequently, more accurate and sensitive techniques are required to make useful measurements at those frequencies.
FIG. 4. (Color online) (a) A typical ocean bubble size distribution presented as the number of bubbles per unit volume per micron radius increment plotted against bubble radius. The slope of the distribution varies from -4 at the larger radii to -1 at the smallest radii. (b) The acoustic attenuation with frequency expected from this bubble size distribution. (c) The same data as (b) with a $\log$ scale on the $y$-axis.
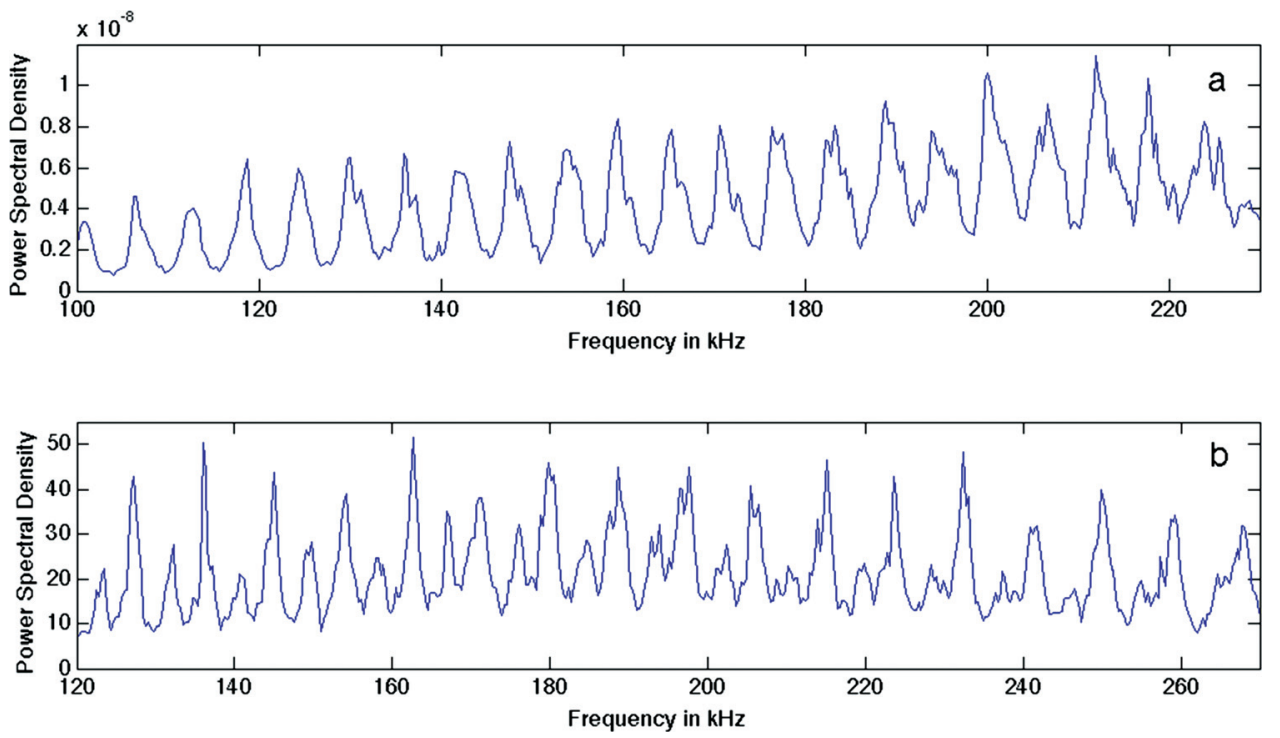

FIG. 5. (Color online) (a) Skewed peaks at frequencies between 200 and $220 \mathrm{kHz}$. In addition, it can be seen that the individual peak heights are fairly variable as frequency increases. (b) An example of alternating low and high peaks, which eventually merge into a single peak at the higher frequencies. Part of the motivation for the research described in this paper was to explore possible reasons for these features. 
each producing an acoustical signal described by the same Green's function. The acoustical pressure waves are reflected back and forth between the steel backing plates, and a steadystate acoustic field is set up. The total detected signal at the receive transducer is the sum of all the attenuated reflections which are incident on that plate, and constructive interference at some frequencies will cause peaks in the measured response while destructive interference causes troughs.

The general method used to describe the physics of this system is to analyze sound making a single traverse across the resonator cavity and being reflected once. Reflection refers to the change of direction of the acoustic signal at the transducer face and may involve a change in amplitude and phase (depending on the thickness and material of the reflecting plate). After a single traverse and a single reflection, the signal is about to start another traverse across the cavity (which will be described by the next iteration in the calculation). To begin with, we will briefly outline the numerical integration method used previously for calculations of the acoustic field ${ }^{6}$ to introduce the much faster matrix method used here.

\section{FORWARD INTEGRATION MODEL}

The full forward acoustic field calculation ${ }^{6}$ used to calculate the eigenvalue $\Gamma$ is computationally very expensive. The transducers are physically identical, so once the signal is in the cavity, it does not matter which one was originally the transmitting transducer and which the receiving. The calculation is carried out for each pass across the cavity, so for clarity here, we will label the transducer that the sound starts from as " 1 " and the transducer that it travels to and is reflected from as " 2 ." The radial pressure distribution leaving plate 1 at radius $r$ is $p_{i}(r)$. After each pass across the cavity, the labels 1 and 2 switch transducers so that the calculation can be carried out for the return journey. The final stage is to multiply this signal by a coefficient that takes into account the physics of the reflection process, so that the output pressure $p_{i+1}(x)$ is the signal at the point when it is about to leave transducer 2 at radius $x$ after one reflection. This integration is repeated until the complex ratio $P_{i+1} / P_{i}$ has converged, and this ratio is taken to be the eigenvalue for that mode. The detected sound pressure field is obtained numerically by integrating the acoustic signal leaving each part of one transducer 1 that arrives at a single point on transducer 2 and then integrating this result across the whole face of the transducer 2 face to get the total received signal. We reproduce Eq. (5) from Farmer et al. ${ }^{6}$ here in order to present the slightly modified formulation which was used in this study and to correct a typographical error in the original paper $[\Re(k)$ was given incorrectly as $k]$.

$$
p_{i+1}(x)=\Psi e^{i \varepsilon} \frac{i \Re(k)}{2 \pi} \int_{r=0}^{X} \int_{\theta=0}^{\pi} p_{i}(r) \frac{e^{i k U}}{U}\left(1+\frac{z}{U}\right) r d r d \theta
$$

where

$$
U=\left[x^{2}+z^{2}+r^{2}-2 r x \cos (\theta)\right]^{1 / 2},
$$

and where $p_{i+1}(x)$ is the pressure distribution at transducer 2 with radial position $x, p_{i}(r)$ is the pressure distribution at transducer 1 with radial position $r, \psi$ is the reflectivity coefficient [given by Eq. (2) in Ref. 6], $\varepsilon$ is the phase shift associated with a reflection [Eq. (3) in Ref. 6], $k$ is the complex wavenumber and $\mathscr{R}(k)$ is its real component, $X$ is the transducer plate radius, $U$ is the path length, and $z$ is the plate separation. Attenuation along the path length adds an imaginary component to the complex wavenumber $k$.

Eigenvalues were calculated at closely spaced frequency intervals by iterations of this integral until $\Gamma$ reached a stable value. The resolution of the numerical integration was increased until the integration results were stable. This value of $\Gamma$ was used to check the results from the faster matrix method shown below.

\section{EIGENFUNCTION ANALYSIS}

The mathematics is most easily described using matrix notation, where a vector $\underline{P}_{i}$ is the radial pressure distribution leaving a transducer face (assumed to have circular symmetry), and $\underline{P}_{i+1}$ is the pressure distribution leaving the opposite transducer after one traverse and one reflection. The elements of $P_{i}$ contain the pressure at equally spaced radius increments across the transducer face. The matrix $\underline{\underline{\Omega}}$ represents the phase change and attenuation of the signal as it leaves one transducer face at a given distance from the center of the transducer and is reflected from another radial distance position on the opposite transducer face. We then get

$$
\underline{\underline{\Omega}} P_{i}=\underline{P_{i+1}} \text {. }
$$

Stable modes exist where the shape of the pressure distribution remains the same after each reflection, so that one or more stable eigenvectors $\underline{P}$ exist:

$$
\underline{\underline{\Omega P}}=\Gamma \underline{P},
$$

where the complex eigenvalue $\Gamma$ represents the attenuation and phase change associated with one traverse of the resonator cavity and one reflection. This is also the eigenvalue described in Ref. 6, in the text accompanying Eq. (7). The total pressure on the receiving plate is therefore the sum of all the reflections. A given signal is reflected twice before it returns to the transducer under consideration, and we assume that the signals are continuous in time so that we can sum all the reflected components to get the final received signal. Equation (8) from Farmer et al. ${ }^{6}$ describes the total signal after $M$ reflections as being proportional to

$$
A=1+\sum_{1}^{M} \Gamma^{2 M} \text {. }
$$

However, the strongest received signal component has already passed across the cavity once from the transmitting transducer when it reaches the receive transducer, so a more appropriate form is a modified version of $A$ called $A_{\text {mod: }}$ :

$$
A_{\text {mod }}=\sum_{0}^{M} \Gamma^{2 M+1}
$$



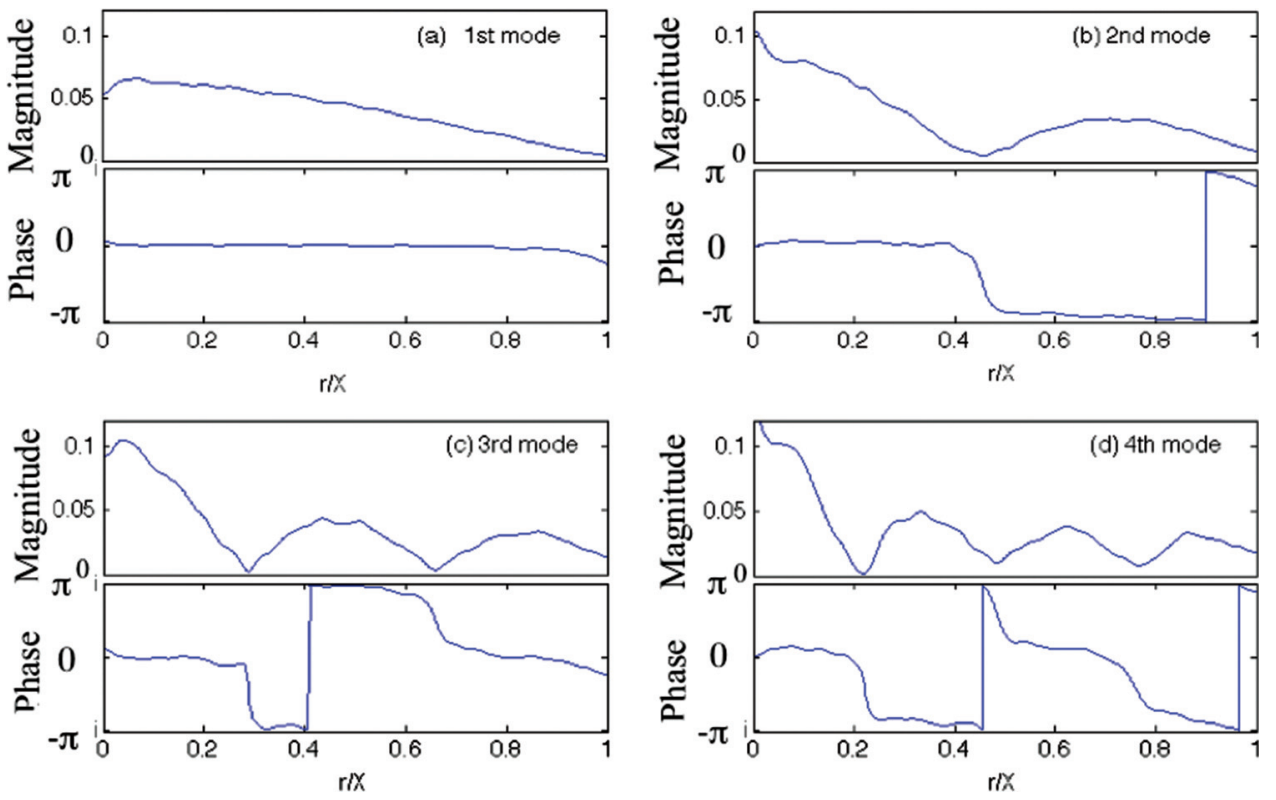

FIG. 6. (Color online) Each pair of panels shows the magnitude and phase of the radial pressure distribution associated with one resonant mode. The first four modes are shown. The top panel in each pair shows the pressure magnitude with frequency, and the lower panel shows the phase variation with radius. Phase shifts of $\pi$ are associated with the magnitude minima. It should be noted that the results shown are the result of calculations at fixed frequency spacings and that the magnitude does not quite reach zero because the chosen frequencies did not coincide exactly with the nodal points. The magnitudes do pass through zero at those points, as shown by the coincident $\pi$ phase shift. and the power spectral density is proportional to $\left|A_{\text {mod }}\right|^{2}$ for $M=\infty$.

Attenuation in the fluid due to bubbles will decrease the magnitude of $\Gamma$ and change its phase slightly, decreasing the resonant peak height and shifting the peak position. Understanding the origin of $\Gamma$ and how to measure it is therefore the key step in inferring attenuation data from a spectrum of resonant peaks. We begin with the full integral model, as presented by Farmer et al., ${ }^{6}$ and then analyze the system by calculating the eigenvalues and the associated eigenvectors using a matrix model.

The matrix notation in Eqs. (4) and (6) provides a compact formulation for this problem and allows for the opportunity to examine modes other than the dominant mode with considerably less computational effort. Each element of the matrix represents the contribution to the acoustic signal at an incremental area (an annulus of radius $r$ and width $\Delta r$ ) on the receiving transducer from an incremental area (an annulus with radius $x$ and width $\Delta x$ ) on the transmitting transducer. The matrix $\underline{\underline{\Omega}}$ was calculated by numerically integrating Eq. (1) for each possible value of the transmitting radius and the receiving radius. The eigenvalues and eigenvectors of the matrix were then calculated using a standard MATLAB routine. The necessary number of matrix elements was determined by comparing the results of the matrix method to the results of the full integration. A matrix size of $500 \times 500$ elements was found to be more than sufficient and was used for this study.

The matrix $\underline{\underline{\Omega}}$ was found to have many physically reasonable modes, and the eigenvectors (representing the radial pressure distribution) are very similar for all resonant frequencies, presumably because the number of wavelengths that fit along a path is a multiple of those for the lowest resonant frequency. The first mode dominates the total resonator response, for reasons discussed below. Figure 6 shows the magnitude and phase of the first four eigenvectors at 440 $\mathrm{kHz}$ for an acoustical resonator with transducers that have a diameter of $0.25 \mathrm{~m}$ and are separated by $0.19 \mathrm{~m}$. These are the radial pressure distributions that will undergo multiple reflections without changing their shape. Without including attenuation, the first four eigenvalues for this system were found to be $0.9966,0.9918,0.9827$, and 0.9704 . The combination of modes that are active depends on the boundary conditions; in this case, the input radial pressure distribution. Each eigenvector is a linearly independent solution, and the input radial pressure distribution provided by the transmitting transducer must be a linear superposition of these eigenvectors. For example, for the case of a uniform input radial pressure distribution, the relative importance of the first five modes is $1.0,0.50,0.33,0.25$, and 0.21 (calculated numerically). So the first mode is dominant, but other modes are active. However, the contributions made by each mode to the detected pressure distribution are not equal.

The PVDF sheet will generate a voltage proportional to the net pressure it receives, and the pressure is not uniform across the transducer surface. The net pressure is proportional to $P(r) d A(r) / A$, where $d A(r)$ is an incremental area at radius $r$ and $A$ is the total area of the transducer face. For the first mode, all the contributions to the pressure across the transducer face are in phase. However, the results in Fig. 6 show that for higher modes a part of the transducer receives a signal that is $\pi$ out of phase with the signal at its centre. To assess the net pressure produced by each phase, we calculate $\sum P(r) 2 \pi r \Delta r$. For the first five modes, the relative contributions (normalized to the contribution of the first mode) are $1.00,0.38,0.23,0.16$, and 0.12 . Combining the relative importance of each mode with its contribution and taking into consideration that the power spectral density is proportional to the square of the net pressure, we see that more than $95 \%$ of the detected resonator response is due to the first mode. The point that we are making in this section is that although the higher order modes could exist, they would have almost no effect on the received acoustic signal because this is necessarily an integration of the pressure response over the whole disc. The response of the PVDF will be positive or negative at particular radii for the higher modes, but the 

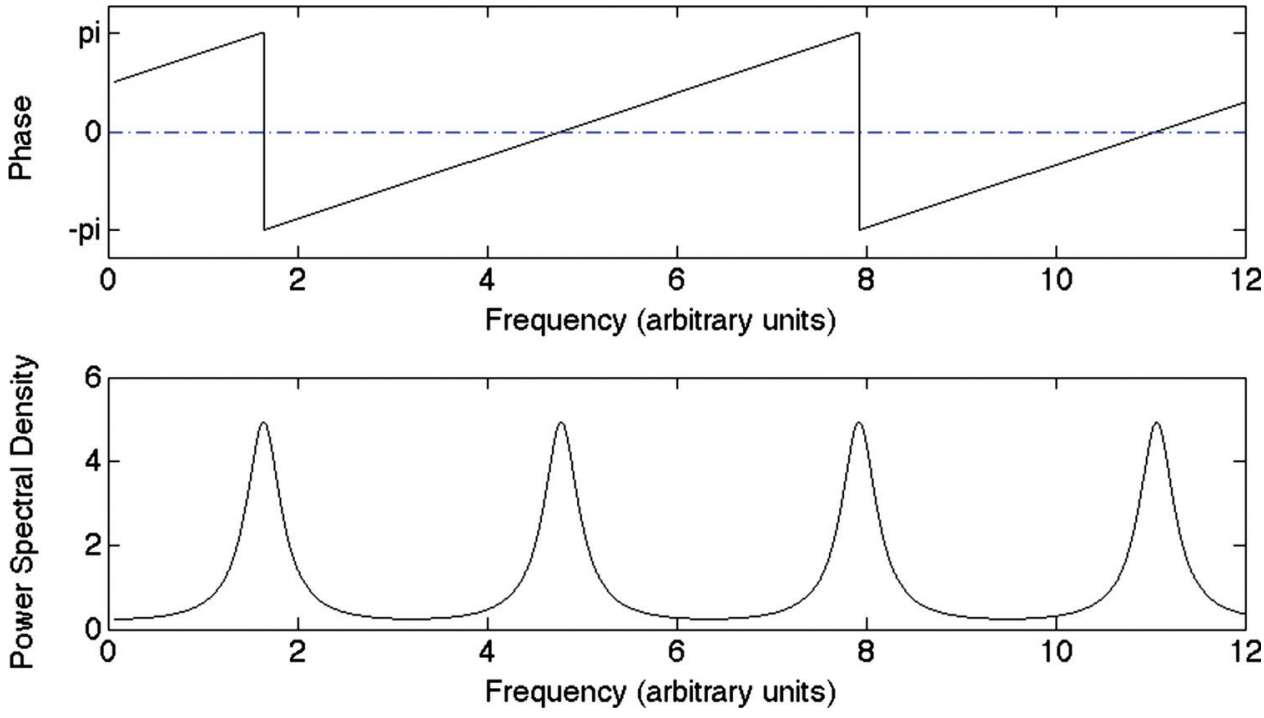

FIG. 7. (Color online) Plot showing the relationship between eigenvalue phase (top plot) and the peaks in the power spectral density (lower plot). Eigenvalue magnitudes change slowly as frequency increases, but the phase changes are rapid. Successive reflections reinforce to form a peak in the spectrum whenever the phase change caused by one cavity traverse is either zero or $\pi$. single output signal from the whole transducer will be a sum of all these effects. We can see that even if those modes are active, their net effect on the received signal is very small. These other modes can only be detected when the quality of the resonance is very high. When $\Gamma$ is very high $(0.95$ or above), the pressure signal is so low between the resonance peaks that peaks due to other resonant modes can be seen in the model calculations. The peaks from other modes have a much lower value of $\Gamma$ so that they are obscured by the dominant mode if there is any overlap. However, none of the peaks produced by the current design of our resonators have an eigenvalue greater than 0.9 . We conclude that the higher modes cannot explain the complex peak shapes seen, and so we continue our analysis assuming that only the first mode needs to be taken into account.

We note that in theory there could also be nonaxisymmetric modes active, while the theory described here only deals with axisymmetric modes. We have ignored the possibility of nonaxisymmetric modes because the resonator transducers have cylindrical symmetry (so only axisymmetric modes are expected) and also because we are unable to detect any modes other than the dominant axisymmetric mode in spectra from our existing resonators.

The final step in the matrix analysis is to predict the spectral response from the calculated eigenvalues. The power spectral density $S$ is proportional to the square of the pressure amplitude [which can be described by the geometrical sum of Eq. (4) when $M=\infty$ ], making the spectral response at any point on the spectrum equal to

$$
S=\left|\left(\frac{K \Gamma}{1-|\Gamma|^{2} e^{i 2 \theta}}\right)\right|,
$$

where $K$ is a constant of proportionality and $\theta$ is the phase of the eigenvalue at that frequency.

At this point, we would like to ensure that the physical meaning of the magnitude and phase of $\Gamma$ is clear. The magnitude of the complex eigenvalue $\Gamma$ is the ratio of the magni- tudes of the signal leaving plate 1 and the signal leaving plate 2 (after one traverse and reflection). Consequently, the situation where $\Gamma=1$ corresponds to zero losses and infinitely perfect resonant peaks. The situation where $\Gamma=0$ represents the case where none of the signal remains after one traverse of the cavity. The phase of $\Gamma$ gives the phase relationship between one reflected signal and the next, and resonant peaks only occur when the phase relationship means that successive reflections reinforce each other. This is important because the magnitude of $\Gamma$ varies only slowly with frequency, but the phase relationship varies relatively rapidly. In our current design of resonator, the phase of $\Gamma$ changes by $2 \pi$ every $8 \mathrm{kHz}$, producing two peaks in every $2 \pi$ cycle. Thus, the change in $\Gamma$ with frequency can be visualized as a rotation in the complex plane, and the total amplitude can be visualized as the sum of successive terms in Eq. (6) in the complex plane.

Figure 7 shows how the variation in the eigenvalue phase with frequency generates the observed peak shapes. These are numerical calculations carried out using the parameters appropriate for our current resonator design. Only the magnitude of the final signal matters for the output, although the phases matter in the sum.

The use of the eigenvalue $\Gamma$ here replaces the traditional quality factor $Q$ because of the cyclical nature of the resonance. When there is a high quality resonance, the magnitude of the signal received at a transducer is very close to the magnitude of the signal transmitted from the other transducer so the magnitude of $\Gamma$ is close to 1 . As the quality of the resonance decreases, there is a limit to the possible frequency width of the resonance because the peaks have a fixed separation in frequency, and as a peak gets wider, it overlaps with the peaks on either side. This limits the possible values of the bandwidth $\Delta \mathrm{f}$ that is needed for the calculation of $Q$. So the traditional definition of quality factor does not apply here for lower quality resonances. However, $\Gamma$ still applies and can take any value between 0 (no resonance at all) to 1 (perfect resonance). A high quality resonance is therefore a peak with a value of $\Gamma$ that is close to 1 . 


\section{THE EFFECT OF BUBBLES}

When a population of bubbles passes between the plates, they change the bulk modulus and attenuate the sound by scattering and absorption, and these effects can be expressed as changes to the real and imaginary components of the complex sound speed.

By using the full forward model of the resonator's acoustic field [Eq. (1)], we confirmed that for a bulk fluid attenuation of $\alpha \mathrm{Np} / \mathrm{m}$, the eigenvalue change is very accurately described by

$$
\Gamma_{\text {attenuated }}=e^{-a z} \Gamma_{\mathrm{o}},
$$

where $z$ is the transducer separation, and $\Gamma_{\text {attenuated }}$ and $\Gamma_{\mathrm{o}}$ are the attenuated and original eigenvalues, respectively. Consequently, if the magnitude of the eigenvalue is known in both cases, the value of the attenuation can easily be calculated. Our approach will therefore be to calculate the eigenvalue for each individual peak and then to use the ratio of the attenuated to unattenuated peak heights to calculate the attenuation. We note that when the ratio of the attenuated peak height to the unattenuated peak height is calculated, the constant $K$ in Eq. (7) cancels out, so there is no need to know the absolute value of the peak height, only the height of the attenuated peak relative to the unattenuated peak.

We start with a calculation of the eigenvalue of the peak observed when no bubbles are present. The eigenvalue associated with a particular resonant peak can be estimated by comparing the spectral level at the troughs and the peak. The corollary of the strong constructive interference at the peak is strong destructive interference at the trough. Therefore, the ratio of the power spectral density at the peak to that at the trough can be used to calculate $\Gamma$, and the higher the peak/trough ratio is, the closer $\Gamma$ is to 1 . Once the initial eigenvalue has been determined, the actual measurement of attenuation is made by comparing the peak heights and is relatively insensitive to the initial $\Gamma$. The advantage of this approach is that it distinguishes between peaks with different resonance qualities (therefore reducing the measurement uncertainty) while still being simple to carry out.

The magnitude of the initial eigenvalue for a peak that can be described by Eq. (7) is then given by

$$
|\Gamma|=\sqrt{\frac{\sqrt{X}-1}{\sqrt{X}+1}},
$$

where $X$ is the peak to trough ratio of the power spectral density peak. This calculation is relatively insensitive to the exact value of $X$ measured-a $10 \%$ change in $X$ results in a change in of less than $2 \%$. The attenuated eigenvalue is then calculated from the ratio of power spectral density peak heights. If the ratio of the attenuated peak height to the initial peak height is $\Phi$, we can calculate the attenuation using Eqs. (7) and (8) to get

$$
\alpha=\frac{-1}{z} \ln \left(\sqrt{\frac{\Phi+\left|\Gamma_{0}\right|^{2}-1}{\Phi\left|\Gamma_{0}\right|^{2}}}\right) .
$$

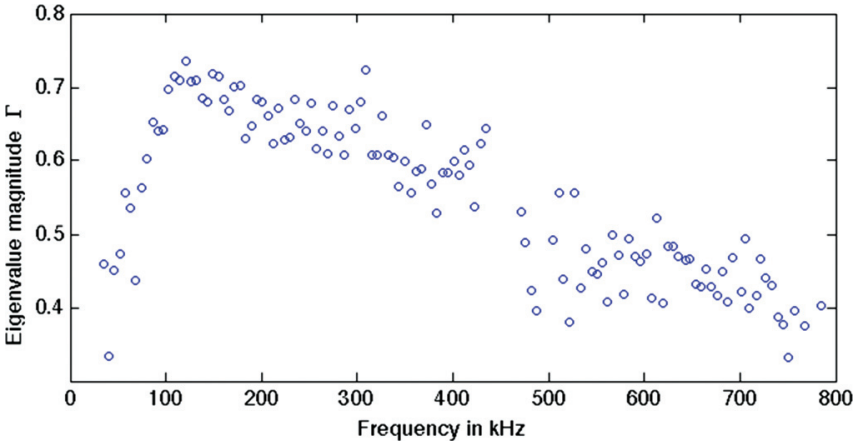

FIG. 8. (Color online) Measured eigenvalue magnitudes for a typical spectrum from one of the current resonators. These are experimental data from an actual resonator, not a modeling result, and this explains the unevenness of the eigenvalue trend with frequency. Each point represents the eigenvalue magnitude of a single peak, measured using the ratio between the power spectral density of each spectral peak and the troughs on either side.

Our procedure is to calculate the initial eigenvalue for each peak on the bubble-free spectrum individually using Eq. (9) and then to store the initial peak height and the eigenvalue magnitude as the reference values. For spectra measured at sea, Eq. (10) is used to calculate the value of attenuation associated with each peak.

For the acoustical resonators that we are currently using, there is a steady downward trend in the initial eigenvalue magnitude with frequency. Figure 8 shows the eigenvalues calculated from the power spectrum produced by one of our current resonators. We emphasize that these are real data and not a modeled result. In this case, at $150 \mathrm{kHz}$, the eigenvalue magnitude is approximately 0.7 and at $800 \mathrm{kHz}$ it is approximately 0.4 , although ongoing engineering efforts are focused on increasing the resonance eigenvalue at all frequencies.

\section{COMPARISON WITH THE PREVIOUS METHOD USED FOR ATTENUATION MEASUREMENT}

The previously used method for calculating attenuation uses a Lorentzian function to model the resonant peaks ${ }^{6}$ and assumes that the half-power bandwidth of any given peak can be calculated from the peak height. By comparing this bandwidth with the half power bandwidth in a bubble-free spectrum, the additional peak width due to the bubble attenuation is inferred.

This approach has several limitations. First of all, it assumes that the spectral peaks have a high quality factor and actually fit a Lorentzian shape. Second, it fails to take into account the periodicity in the spectrum, which results in consecutive peaks starting to touch each other as the spectral quality decreases. Figure 9 shows the best fit of a Lorentzian shape to spectral peaks with different eigenvalues. Although the fit is good for an eigenvalue of 0.8 , there is a significant deviation when the eigenvalue is 0.5 . Finally, the approach provides no flexibility in accounting for irregular peak shapes. By contrast, the method presented in this paper is based on the more detailed physics of the resonant process and can account for all of these features. 

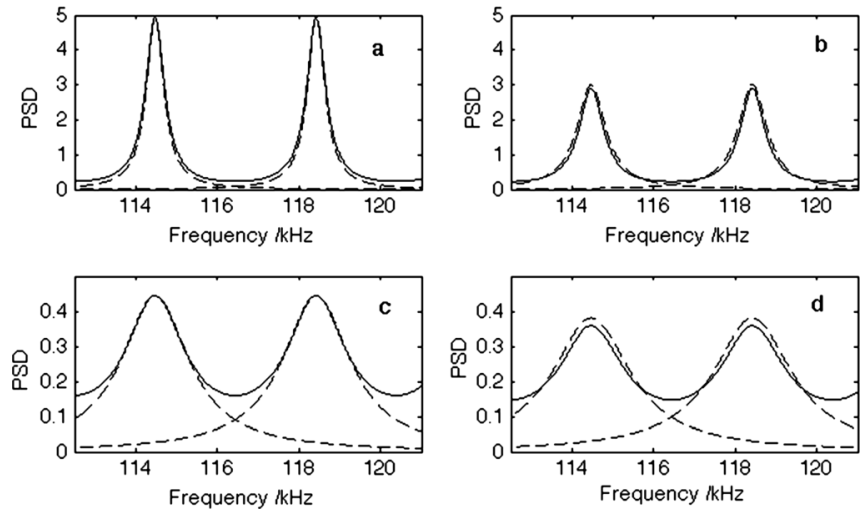

FIG. 9. A comparison of spectral peaks calculated using the matrix model (solid lines) with the best fit Lorentzian peak shapes (dashed lines). (a) and (b) both show peaks with an initial eigenvalue of 0.8 and (c) and (d) show peaks with an initial eigenvalue of 0.5 . The peaks in (b) and (d) have both been attenuated with the same absolute attenuation. The Lorentzians shown here are used to estimate the attenuation. When (b) is compared to (a), the error in the Lorentzian result is $4.3 \%$. When (d) is compared to (c), the error in the Lorentzian result is $45 \%$.

Figure 10 shows the percentage error in the Lorentzian approach when compared to the matrix approach presented here for a range of initial eigenvalues. The Lorentzian method always provides an overestimate. The error in the Lorentzian method is less than $10 \%$ for peaks with an eigenvalue above 0.7 but fails to work at all for eigenvalues below 0.4 (since the peak is no longer more than twice the trough value). As the eigenvalue magnitude decreases below 0.7 , the error associated with the Lorentzian method increases rapidly.

An examination of the spectra published by Farmer et $a l .{ }^{6}$ suggests that most of the spectra were associated with eigenvalue magnitudes between 0.6 and 0.7 . This suggests that there may have been a systematic error in the absolute value of the measured attenuation between $10 \%$ and $20 \%$, but this is relatively small compared to the general variability of oceanic measurements. However, it is possible that a systematic frequency dependent error could have more influence when estimating the slope of the bubble size distribution. To determine the extent of this effect, we considered a hypothetical spectrum with eigenvalue magnitudes which varied linearly from 0.8 at $50 \mathrm{kHz}$ to 0.52 at $400 \mathrm{kHz}$. This is a variation that is similar to that on the spectrum shown in Figure 9(a) of Farmer et al. ${ }^{6}$ Calculations were carried out for bubble distributions with populations that were proportional to $a^{v}$, where $a$ is bubble radius, and $v$ is the exponent of interest. For values of $v$ from -1 to -4 (a typical range for bubble population measurements beneath breaking waves), the slope obtained using the Lorentzian method underestimated the true slope by approximately 0.1 . Because this is a relatively small error when considering oceanic bubble size distribution models, the general results of Farmer et $a{ }^{6}{ }^{6}$ and other papers published using resonators below $200 \mathrm{kHz}^{8,9}$ are not significantly affected by the processing method used. However, the new matrix-based method presented here provides a straightforward basis for analyzing spectral peak heights to infer attenuation and is required for measurements at higher frequencies.

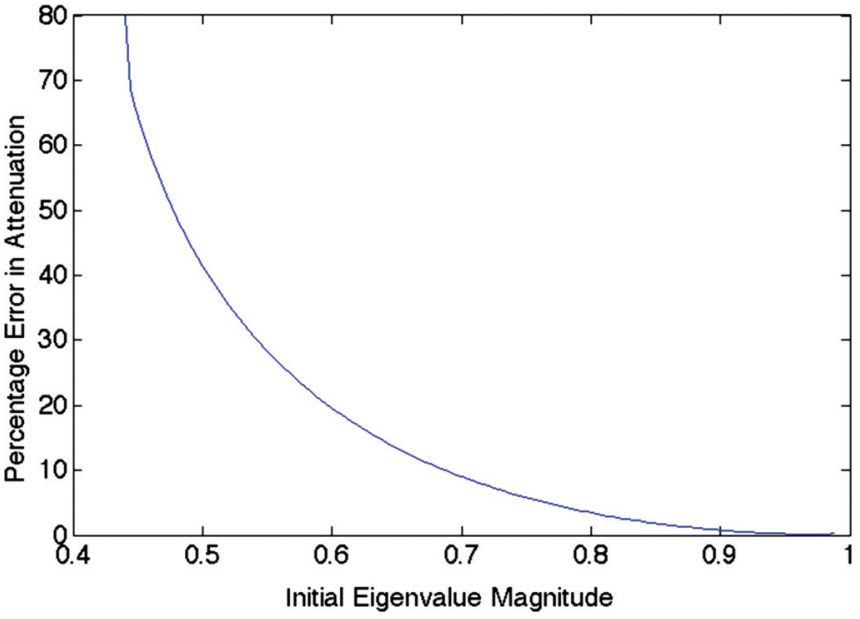

FIG. 10. (Color online) The percentage error caused by using the Lorentzian method plotted against the magnitude of the initial eigenvalue $\Gamma$.

\section{FLEXIBILITY IN THE RESONATOR MODEL}

One of the scientific objectives behind the development of this new technique was to obtain an understanding of the spectral features and irregular peak shapes that have been observed using acoustical resonators. For example, several of our resonator systems have produced spectra with alternating low and high peaks in the region from 100 to 400 $\mathrm{kHz}$, gradually changing so that the larger peak in each pair dominated completely at higher frequencies (see Fig. 11) and double-spaced peaks were produced. Based on the model developed in this paper, we suggest that this happens because of electrical cross talk between the transmit and receive transducers. Cross talk would cause some of the transmitted signal to be added directly to the output signal of the receive transducer and consequently successive peaks would alternate in size. To understand the consequences of possible cross talk, we note that the peaks should occur when the round trip path length from one reflection off the receive transducer to the next (two traverses of the cavity) is equal to an integer number of wavelengths. This corresponds to an integer number of half-wavelengths for one traverse of the cavity, so if at one peak the reflections off the two transducers are in phase, at the next peak these reflections are $\pi$ out of phase. Cross talk would result in adding the signal at the two transducers, and so peaks corresponding to the inphase case would be reinforced while peaks corresponding to the out-of-phase case would be partly cancelled. If the cross talk is the majority of the signal, peak spacing will double because a peak will only occur when an integer number of wavelengths fit into a single path length from one transducer to another. We note that using a conducting wire to permit direct electrical contact between the resonator back-plates (artificially facilitating cross talk between the transmit and receive plates) generates this alternating peak effect in frequency ranges where it was not previously present. This supports the hypothesis that cross talk could be responsible for the alternating peaks seen when the resonator is in normal operating mode.

This effect was modeled by summing the pressure at the receiving transducer and a fraction $C$ of the pressure at the 

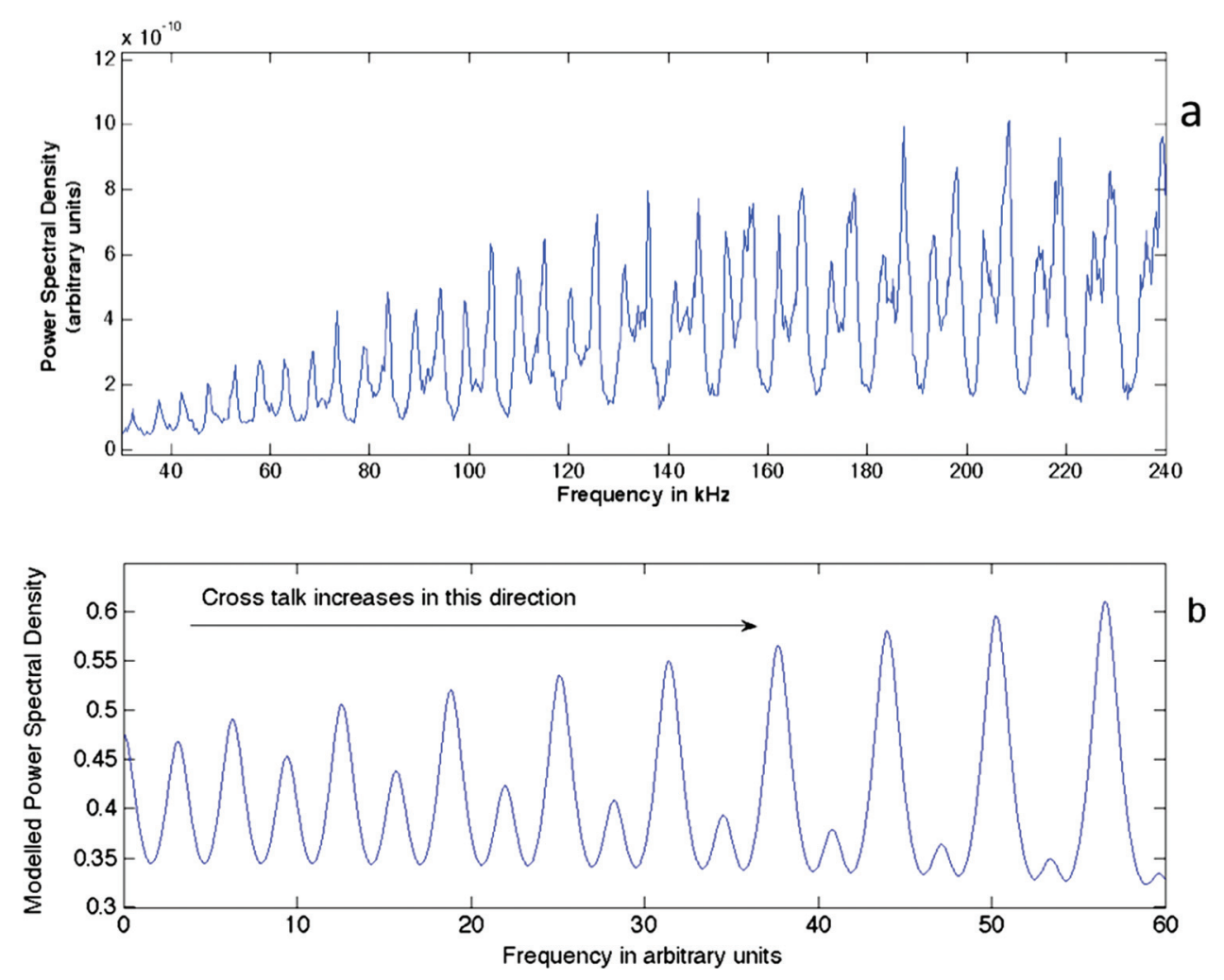

FIG. 11. (Color online) An unexpected feature that was produced by some resonators was the transformation with frequency from singlespaced peaks to double-spaced peaks, shown in (a). The corresponding modeled response is shown in (b), as discussed in the text and described by Eq. (11). The cross talk coefficient $C$ increases as the arbitrary frequency increases. transmitting transducer. The resulting amplitude including the effects of cross talk $A_{\mathrm{CT}}$ can be given by

$$
A_{\mathrm{CT}}=\frac{|\Gamma|}{1-|\Gamma|^{2}}+\frac{C}{1-|\Gamma|^{2}},
$$

where $C$ is a frequency-dependent parameter that depends on the extent of the cross talk. Figure 11(b) shows the power spectral density predicted by this model as the value of $C$ increases with frequency. Qualitatively this produces a spectrum with a similar shape to 11(a). The actual value of $C$ to use in practice at any given frequency can be determined by fitting the model to a bubble-free spectrum. We have fitted this model to such a spectrum for one of our resonators and have found that attenuation could still be measured from these double peaks using Eq. (11). The engineering aim is to manufacture resonators that do not produce this alternating peak height, but if it is present Eq. (11) means that the attenuation measurements can still be made. In addition, this theory for the cause of the alternating peaks feature may advance attempts to eliminate it.

\section{CONCLUSIONS}

We have outlined an improved method for interpreting the output of an acoustical resonator to measure the additional acoustical attenuation caused by changes in the bulk properties of the liquid between the transducer plates. In contrast with the methods previously used, this method is based on the specific physics of the resonance process in an open-sided acoustical resonator. A matrix which describes the propagation of ray paths across the resonant cavity has been calculated, and the eigenvectors of this matrix correspond to stable resonant modes. Consideration of the likely contribution of the first few modes leads us to conclude that only the first mode needs to be taken into account when calculating the spectral response of the resonator system. The magnitude and phase of the eigenvalues for this mode can be used to calculate the spectral response of the resonant system, and changes in the peak heights can then be used to estimate changes in attenuation. This model for resonator performance is more flexible than the method used previously and can account for more realistic spectral shapes. This does not invalidate the previous results gathered using acoustical resonators, although the analysis method may have caused a systematic error of the order of $10 \%-20 \%$ in the attenuation measurement in the frequency range from 10 to $200 \mathrm{kHz}$ and a similar error in bubble void fraction estimation. However, for measurements which include higher frequency resonant peaks (including the measurements up to $1 \mathrm{MHz}$ discussed here), the method proposed here significantly improves the accuracy of the results.

\section{ACKNOWLEDGMENTS}

This research was carried out as part of the $\mathrm{RaDyO}$ program (Radiance in a Dynamic Ocean) and was funded by the Office of Naval Research (Contract Nos. N000140610072 and N000140610379) and Fisheries and Oceans Canada.

${ }^{1}$ O. Diachok, "Absorption spectroscopy: A new approach to estimation of biomass," Fish. Res. 47, 231-244 (2000).

${ }^{2}$ J. W. Caruthers, S. J. Stanic, P. A. Elmore, and R. R. Goodman, "Acoustic attenuation in very shallow water due to the presence of bubbles in rip currents," J. Acoust. Soc. Am. 106, 617-625 (1999). 
${ }^{3}$ S. Vagle and D. M. Farmer, "The comparison of four methods for bubble size and void fraction measurements," IEEE J. Ocean. Eng. 23, 211-221 (1998).

${ }^{4} \mathrm{H}$. Medwin, "Acoustical determinations of bubble size spectra," J. Acoust. Soc. Am. 62, 1041-1044 (1977).

${ }^{5} \mathrm{~N}$. Breitz and H. Medwin, "Instrumentation for in situ acoustical measurements of bubble spectra under breaking waves," J. Acoust. Soc. Am. 86, 739-743 (1989).

${ }^{6}$ D. M. Farmer, S. Vagle, and A. D. Booth, "A free-flooding acoustical resonator for measurement of bubble size distributions," J. Atmos. Ocean. Technol. 15, 1132-1146 (1998).

${ }^{7}$ D. M. Farmer, S. Vagle, and A. D. Booth, "Reverberation effects in acoustical resonators used for bubble measurements," J. Acoust. Soc. Am. 118, 2954-2960 (2005).
${ }^{8}$ S. Vagle, D. M. Farmer, and G. B. Deane, "Bubble transport in rip currents," J. Geophys. Res. 106, 11677-11689 (2001).

${ }^{9}$ J. R. Gemmrich and D. M. Farmer, "Near-surface turbulence in the presence of breaking waves," J. Phys. Oceanogr. 34, 1067-1086 (2004).

${ }^{10}$ D. Stramski, E. Boss, D. Bogucki, and K. J. Voss, "The role of seawater constituents in light backscattering in the ocean," Prog. Oceanogr. 61, 2756 (2004)

${ }^{11}$ X. Zhang, M. Lewis, M. Lee, B. Johnson, and K. Korotaev, "The volume scattering function of natural bubble populations," Limnol. Oceanogr. 47, 1273-1282 (2002).

${ }^{12} \mathrm{O}$. R. Hughes, "Frequency dependence of hysteresis associated with the electromechanical performance of PVDF film," J. Polym. Sci., Part B: Polym. Phys. 45, 3207-3214 (2007). 